\title{
Opinion Sentiment Analysis
}

\author{
Alok Kumar, Renu Jain \\ University Institute of Engineering and Technology, Kanpur, India
}

\begin{tabular}{l} 
Article Info \\
\hline Article history: \\
Received Jun 19, 2016 \\
Revised Aug 14, 2016 \\
Accepted Aug 22, 2016 \\
\hline
\end{tabular}

\section{Keyword:}

Aspect extraction Feedback analysis Natural language processing Opinion mining

Sentiment analysis

\begin{abstract}
An automatic system to analyze the opinions/feedbacks of the people working in any organization is proposed. The proposed system does two major jobs: the first part extracts the most relevant/important aspects from the textual feedbacks provided by clients/managers/co-workers for all the employees having similar job profile and the second part measures the degree of positivity or negativity for all the extracted aspects for every employee and then an overall performance of everyone is evaluated. We have implemented the system to study the feedback of teachers. The proposed system is flexible and versatile than the existing feedback evaluation system of teachers where students evaluate the teachers on the predefined aspects decided by experienced and senior teachers. Our system, Faculty Evaluation System (FES) identifies strengths and weaknesses of teachers on all aspects which are important to students. This information may be used by higher authorities of the institute in identifying suitable faculty members for different academic and administrative activities of the institute in addition to the teaching responsibility.
\end{abstract}

Copyright $(0) 2016$ Institute of Advanced Engineering and Science. All rights reserved.

\section{Corresponding Author:}

Alok Kumar,

Department of Computer Science and Engineering,

University Institute of Engineering and Technology,

Kanpur, India.

Email: akumar.uiet@gmail.com

Mob:+919307071522

\section{INTRODUCTION}

We, human beings always make our decisions on the basis of the information gathered directly or indirectly whether we buy a product or we hire a person or we need to get some specific job done. When we want to purchase a product, we look for few very specific features in addition to the basic features within certain financial constraints before making the final decision. But when we deal with human beings, it is very different. Every human being possesses many dimensions having specific characteristics for each one. For example: A teacher may be a very knowledgeable and friendly person but from students' point of view he/she may not be a proper teacher. Hence, analyzing the feedback of humans is far more complicated than analyzing the feedback of inanimate things or it can be said that human behavior is more contexts dependent and we need to evaluate them in every context separately.

Here, we are focusing on analyzing the feedback of human beings given by human beings. The objective of this paper is no just to evaluate the feedback on some pre-defined aspects and provide ratings on that basis. Instead, we are extracting the aspects from user feedbacks itself which users think are important from their point of view and then evaluate the degree of positivity and negativity for each aspect. We have used linguistic features and a combination of machine learning techniques to extract aspects and measure the degree of existence of each aspect for every individual for whom data has been collected. A system called FES for analyzing the teachers' feedback has been implemented.

Traditional teacher's feedback evaluation system is a questionnaire based system. In this system, a form (questionnaire) is given to each student. Questionnaire has a number of questions related to the teacher 
or subject. Student gives numeric score or ordinal score (i.e. below average, average, good, excellent) to each question included in questionnaire. On the basis of responses of all students, it is determined how much a teacher is able to contribute in his/her course. The main problems of questionnaire based system are:

a. Deciding the question set is a difficult task. There is no standard method or technique to decide the questions for a questionnaire. Higher authorities decide questions on the basis of their personal experiences only.

b. Traditional questionnaire based system becomes very restricted. Students can assign scores only on those questions which are mentioned in questionnaire. There is no provision for students to assign score on other aspects of teachers which may be more important to them.

A teacher despite having a very good command over the subject may have some vital teaching qualities that motivate the students for learning or some repulsive way of teaching while delivering a lecture, these known and unknown important features can by identified by the students only. For instance, the way a teacher introduces a new topic, his/her gesture in the class, writing skills, etc may be more important to a student than the details in which the teacher has completed the course. By determining teachers' aspect, we can use it for many academic and administrative purposes as mentioned below.

a. Aspect grading: In existing system, we assume that each question/ aspect included in questionnaire has equal weightage for example presentation and knowledge of a teacher but this is actually not true. Some aspects are more important with respect to other aspects. For an example, presentation is more important aspect than knowledge. With the help of our proposed system, we can grade the aspects of a teacher or we can say that importance of aspects can be determined. Aspect grading is helpful for the higher authorities during the selection of teachers as well as during the measuring effectiveness and usefulness of teachers.

b. Committee Formation: For smooth running of an institute or department, a number of committees and cells are formed by the head of institute or by the head of department. Each committee is responsible for some specific task and number of faculty members are required who are suitable for a committee. For discipline committee, we require a number of teaches who are strict in nature while for a counseling cell, we require a number of faculty members who are cool in nature. Our proposed system is useful to identify suitable persons for such committees.

c. Impact of Research and Training: Institute assumes that research and training improve the effectiveness of teachers. All institutes always encourage their faculty members for research and training without knowing the impact of it on the effectiveness of teacher in class room. Our proposed system will help in identifying the impact of research and training on teachers.

d. Impact of Administrative Duties: Apart from teaching, institute assigns additional duties to teachers. With the help of our proposed system, we can identify impact of additional assignments on teacher's effectiveness in class room.

e. Demographic Analysis: With our proposed system, we can perform age and gender based analysis of teacher's features. Inferences of such demographic analysis will be helpful for higher authorities to choose suitable gender based and age base selection of faculty members of some specific task.

To measure the effectiveness of a teacher to the satisfaction of every student, we are proposing an aspect based sentiment evaluation system. In the proposed system, we collect student feedback in the form of running text. Using linguistic features and machine learning techniques, we have analyzed the students' opinion for each teacher for all important aspects extracted from the data itself. This evaluation model overcomes the above mentioned problems and in addition administration will also get information about each teacher on a variety of aspects which can be further used by authorities for assigning important responsibilities to them. During the aspect identification process, the system chooses the vital aspects by grading each aspect on the basis of feedback of students. Aspect detection and evaluation of each aspect for every faculty/employee allows an organization to use this information for effective utilization of its people leading to better growth.

We divide our research paper in six sections. Related works are discussed in section 2 . The section 3 highlights the differences between existing sentiment analysis systems and our system implemented for evaluation of teachers' feedback. Architecture of the proposed system and functions of sub modules are explained in section 4. Results with conclusions are discussed in section 5.

\section{RELATED WORK}

$\mathrm{Hu}$ and Liu [1] proposed a method to perform aspect based sentiment analysis of products reviews. In this paper, authors assume that the product aspects are nouns and noun phrases. Nouns and noun phrases are extracted using association rule mining algorithm of data mining. Each sentence of review is considered as a transaction. This method is quite simple and has been used by many researchers with some modifications. Popescu and Etzioni [2] used point wise mutual information gain (PMI) to check whether a noun or a noun 
phrase is a valid aspect or not. Authors computed PMI between candidate aspects and meronymy discriminators of product. Meronymy discriminators of product "camera" are 'of camera', 'camera has', 'camera comes with'. PMI (w1, w2) is the ratio of joint concurrences of word ' $w 1$ ' and word ' $w 2$ ' with respect to the individual occurrences of word ' $w 1$ ' and 'w2'. Only those candidate aspects are considered valid aspects whose PMI score is greater than threshold value. Threshold value is determined experimentally.

Qiu et al. [3] proposed a double propagation algorithm to extract new aspects and sentiment expressions on the basis of syntactic structures of aspects and sentiment expressions. They use the bootstrapping technique where they start with some seed sentiment words and on the basis of known seed words new aspect are identified and vice versa.

Jin and Ho [4] consider aspect based sentiment analysis as a sequence labeling problem. Authors manually tag each word of reviews with appropriate labels and impose lexicalized Hidden Markov Model and from the tagged sentences, system learns patterns to identify aspects and sentiment expressions.

Jakob and Gurevych [5] extend the approach suggested by Jin and Ho [4] using Conditional Random Fields algorithm (CRF). CRF is learned in one domain and used to extract in other domain.

Mei et al., [6], proposed a topic model based approach which consists of an aspect model to determine aspects of the products and then positive and negative sentiment model determines the polarity of extracted aspects.

A. Kumar and R. Jain [7], proposed a system architecture to perform sentiment based analysis of teachers' feedback given by the students in textual form. Authors ignored objective sentences and identified the essential aspects of teacher by using conditional maximum entropy based approach with mutual information and TF-IDF of respective words in subjective sentences. Sentiment score is assigned to all identified aspects on the basis of point wise mutual information gain between identified aspects and sentiment words.

Ding, Liu and Yu [8], proposed a sentiment lexicon based method to determine sentiment polarity of aspects in sentences. Score +1 and -1 is assigned to all sentiment word belonging to positive and negative lexicons respectively. Sign of sentiment words are inverted if sentiment shifter is present near to sentiment words. Conjunctions of phrases are handled carefully to decide the sentiment of aspects. Finally an aggregation function is used to aggregate sentiment score of mentioned aspects.

A formal grammar based system to decide sentiment polarity of simple and compound sentences [912]. Formal grammar rules are expressed in form of BNF. Overall sentiment is computed on the constitutional words and syntactic structure with other words. Formal grammar is in generalized form, it can be used in any domain with some modifications.

\section{DIFFERENCES BETWEEN EXISTING SENTIMENT ANALYSIS SYSTEMS AND OUR SYSTEM}

The methodology used in our opinion sentiment analysis system differs from existing systems in many ways. Most of the research in the field of sentiment analysis has been around to perform aspect based sentiment analysis related to products, services, movies etc taking the consumer feedback. The focus of the analysis is to enhance the product quality, its features and eventually the sales. Most of the product features built are well understood according to the need of common users and some specific features are kept for a small group of consumers. But, in case of human beings, a multi-dimensional skill set is required to fulfill the requirements of a position in an organization. With humans, many qualities are taken for granted and sometimes while giving opinions many of us do not even mention those explicitly. Not much work as on date is available in this regard.

We implemented sentiment analysis for human beings and examined that non living things i.e. products or services generally have features that are in the form of nouns and compound nouns. Sentiment analysis systems capture the aspects from the feedbacks related to products or services through the noun syntactic category. In contrary to this from our study of sentiment analysis for professionals/ individuals, we observed that not only nouns and compound nouns but other syntactic categories like adjectives, adverb and sometimes even verbs may play an important role in providing aspects for sentiment analysis. For example: let us look at the sentences 'he is a very interactive and frank teacher' and 'she takes her teaching job very sincerely and understands the problems of students'. Here we observe that adjectives 'interactive' and 'frank' in the first sentence and adverb 'sincerely' and verb 'understands' in the second sentence are valid metrics/aspects of teachers. We can therefore very clearly see that the approach for handling the sentiment based feedback for objects / services and handling the feedback for professionals will not be same. It will be more complicated and difficult to extract the aspects from data for human beings.

Traditionally, questionnaires are used to take the feedback of the employees, teachers, etc from customers or students so that processing of data is easier. As textual feedback allows us to give liberal views without any bar, processing of such data is tedious and it becomes almost impossible to grade them objectively. The freedom to express views in textual form allows the users to express their views for any aspect which may be more relevant according to them. In most of the existing systems, the aspects of the item/product mentioned 
in each sentence of the feedback along with its degree of affirmation are captured and then for each aspect the positivity of the product is evaluated. But in case of humans, this approach may not give the correct degree of affirmation or negation for the identified aspects as the list of important aspects mentioned in each feedback may vary from user to user and in some cases the lists may be almost complimentary. In our system, we assume that each feedback is written taking into account all possible aspects but the user expresses his/her opinions for only those aspects which are either very important to them or which have high degree of positivity/negativity. The logic behind our argument is that generally we, human beings do not talk about the obvious aspects/traits present in human beings or those which are present in average degree i.e. aspects which are normal in degree. In our proposed system, first we extract the list of all possible aspects from all feedbacks and then categorize those to be either above average, below average or average. If the system finds that a feedback has not mentioned of an identified aspect, system assigns 'average' degree of freedom to it. This way it is considered that every user has opinion about every aspect but he/she may not express it explicitly in the feedback.

When a user writes feedback about an item, he/she writes about those aspects only which seem important to him/her and therefore, while assigning the sentiment score for that user, existing systems evaluate sentiment score for the mentioned aspects only. Many of the aspects remarked in two different feedbacks may be entirely different and it makes difficult to compare the two feedbacks. While in our proposed system, we assign sentiment score to all possible aspects of item found in all the feedbacks whether a user has given his/her remarks for that or not. Above average or below average sentiment score is assigned to remarked aspects and it is computed from respective sentences. All other aspects which are not remarked in the feedback by the user are assigned average sentiment score. With our system, we can compare items (products / services / human) with respect to other items as well as two feedbacks can be compared.

\section{PROPOSED SYSTEM}

The proposed feedback analysis system takes collection of feedbacks in the form of running text for all the teachers of an institute or employees of an organization. In the first phase, it identifies the possible aspects and then selects essential aspects on the basis of the pre-decided threshold and then in the second phase, it evaluates the performance of each employee taking into account the essential aspects. We follow the modular approach to design our system. We have divided our system into three modules namely Aspects Retrieval, Aspect Sentiment Analyzer and Teacher Evaluation Reports [13-18]. Every module is further divided into sub modules. We make use of natural language processing and sentiment analysis techniques to implement the modules of our system. The basic three tire architecture of sentiment based feedback analysis system is mentioned below in Figure 1.

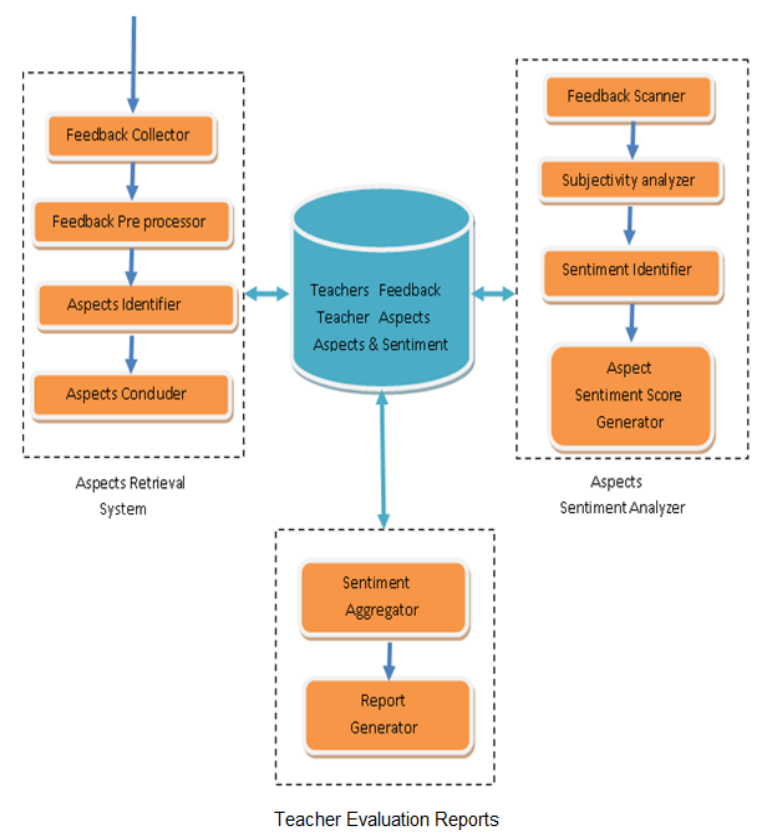

Figure 1. Architecture of Proposed System 


\subsection{Aspect Retrieval Module}

This module collects and combines the students' feedback from different sources and identifies all essential aspects of teachers (liked or disliked by the students). This module is further divided into following sub modules.

\subsubsection{Feedback Collector}

This sub module collects feedback of teachers from the specified sources and stores feedback in database, sources may be remote or directly from the organization. We collected feedback from the following sources.

i. $\quad$ www.ratemyprofessor.com

ii. www.myfaveteacher.com

iii. Textual feedback of 10 teachers of our college from 180 engineering students.

\subsubsection{Feedback Pre Processor}

Feedback collected by the feedback collector module is in raw form. Collected raw feedback is processed by this module to remove unwanted ASCII characters, removal of unwanted text like HTML tags, images, titles, hyper links etc. Misspelled words are corrected by using the auto correction library of NLTK. Another important tasks of this module is text normalization, removal of named entities i.e. subjects name, institute name, teachers name etc.

\subsubsection{Aspects Identifier and Aspects Concluder}

These are the most important sub modules of our proposed system. Input to these modules is pre processed feedbacks of all teachers and output is a list of aspects of teachers (liked and disliked by the students). Aspect Identifier module recognizes all possible aspects of teachers from student feedbacks and Aspect Concluder module mines only essential aspects from possible aspects which are relevant in current context.

We have used a semi supervised approach to implement the Aspects Identifier and the Aspects Concluder modules. Our proposed system is based on $\mathrm{Hu}$ and Liu [1] approach. As mentioned in [1], we assume that the possible teachers' aspects are nouns, noun phrases, compound nouns and in addition to that as discussed in section 3, we also check for some adjectives and adverbs like knowledgeable, conceptual, strong, friendly, encouraging etc. So, we first find all possible aspects (nouns, noun phrases, compound nouns and adjectives, adverb) from all collected feedbacks. Processing of all feedbacks is done so that every aspect is observed by the students is not left but there may be many irrelevant words/phrases in all possible aspects. To remove such irrelevant words, we choose some well known aspects as seed aspects and add to the final list of aspects [19, 20]. We assume that valid teachers' aspects have similar context, or we can say aspects are paradigmatically related words. We iteratively increase the aspects list either choosing contextually similar aspects or frequently occurred words having sentiments_and the process is continued until all possible new aspects are added to final list of aspects. Steps of our proposed system are given below.

Algorithm:

Input: Feedbacks given by the students

Output: List of important aspects for teachers

Step 1: Extract all nouns, noun phrases and compound nouns in all the feedbacks collected from the students and add to the possible_aspects_list.

Step 2: Add all modifiers (adjectives and adverbs) words of 'teacher', 'institute' and 'subjects' ( except usual common modifiers like good, bad, awesome, worst etc) to the possible_aspect_list.

Step 3: Add some well known features of teachers to the final_aspects_list as a seed features.

Step 4: Compute the contextual information of all words /phrases present in final_aspects_list.

Step 5: Compute the contextual information of all words /phrases present in possible_aspects_list.

Step 6: Convert contextual information of words / phrases presented in possible_aspects_list and final_aspects_list in the form of vectors.

Step 7: Measure contextual similarity of each word/phrase in possible_aspects_list to the words/phrases in final_aspects_list.

Step 8: Add highly contextually similar words of possible_aspects_list to final_aspects_list if words frequently associated with sentiment words.

Step 9: Repeat step 2 to 8 until new words/phrase are added to the existing final_aspects_list.

Step 10: Remove all words final_aspects_list whose frequency is less than threshold value. Frequency threshold value is decided experimentally. 
Step 11: Remove invalid aspects (words / phrases) from final_aspects_list using linguistic and domain knowledge. Remaining words / phrases in final_aspects_list are the essential aspects for the teachers.

\subsection{Aspects Sentiment Analyzer}

We assume that students give feedback on all aspects of teachers which are identified by the Aspects Retrieval module but students mention only those aspects in his / her feedback which are either below average or above average, aspects of average level are not highlighted by the students. We divide Aspect Sentiment module in the following sub modules.

\subsubsection{Feedback Scanner}

This module processes feedbacks one by one and populates two lists of aspects. First list has the aspects mentioned by a student in his/her feedback taking into account those sentences only which have subjective data. To check whether a sentence is subjective or objective, it is passed to Subjectivity Analyzer module. Second list has aspects which are not mentioned in a feedback. Both lists contain only those aspects which are finalized by Aspects Retrieval module. Sentiment score of aspects in both lists are calculated later.

\subsubsection{Subjectivity Analyzer}

There are two types of sentences in the feedbacks given by the students: subjective sentences and objective sentences. Objective sentences have some factual information, in these types of sentences; students do not highlight any aspect. For example: 'He is the Head of the Department', 'He is teaching us Computer Network.' In subjective sentences students remark on his/her likes or dislikes of that teacher's aspects. Our system processes only subjective sentences and ignores objective sentences.

To identify subjective sentences, we have used naïve based approach to solve it assuming a two class classification problem. Input to the system is a training set. In training set, all the sentences are labeled with appropriate label i.e. subjective or objective. On the basis of input examples, Naïve Bayes algorithm generates probabilistic distribution of words for each class. On the basis of probabilities of words in different classes, it is determined whether the sentence is subjective or objective.

\subsubsection{Aspect Sentiment Identifier}

This module considers feedback of one student at a time; it explores this feedback for each of the possible aspects. There would be three possibilities.

a. Aspect is present in the feedback and is having positive orientation/polarity. Such an aspect is assigned above average score.

b. Aspect is present in the feedback and is having negative orientation/polarity. Such an aspect is assigned below average score.

c. Aspect is not present in the feedback and such an aspect is assigned a pre-defined average score.

Likewise feedback provided by all the students is processed for aspect sentiment identification. To implement Aspect Sentiment Analyzer, we have proposed a novel algorithm. Proposed algorithm assigns sentiment score to all aspects with respect to each feedback whether aspects are mentioned in the feedback or not. Mentioned aspects in the feedback are assigned positive or negative sentiment score. To compute sentiment scores of aspects, we need to classify the the words as positive or negative according to the semantic weightage in the sentence. Many lexicons in open domain are available which provide sentiment scores but to get more context dependent sentiment scores, we have developed domain specific teacher sentiment lexicon to get better sentiment scores for the aspects using bootstrapping technique. Steps of our proposed algorithm used in Aspect Sentiment Analyzer are given below.

\footnotetext{
Algorithm:

Input: Feedbacks given by the all students, List of Aspects provided by Aspects Retrieval module, Teacher Sentiment Lexicon.

Output: Assign a sentiment score to each aspect.

Step 1: Select a feedback.

Step 2: Read the next sentence from the selected feedback.

Step 3: Pass the sentence to Subjectivity Analyzer module to determine whether the sentence is subjective or objective.

Step 4: If the sentence is objective then ignore it and go to the step 2, otherwise go to the step 5.

Step 5: Look for the aspects in sentence and match it with the list provided by the Aspects Retrieval module. If aspects match with the list provided by the Aspects Retrieval module then go to step 6 otherwise go to step 2 .
} 
Step 6: If the part of speech of the identified aspect is adjective or adverb and it is present in positive lexicon then assign sentiment label 'above average' to the identified aspect otherwise assign 'below average' sentiment label. However, if any sentiment shifter like not, never, do not etc are present then final sentiment score is assigned by our sentiment shifter function.

Step 7: If the part of speech of identified aspect is noun or noun phrase, extract nearby adjective as a sentiment word. If extracted adjective is present in the positive lexicon then assign sentiment label 'above average' to the identified aspect otherwise if the extracted adjective is present in negative lexicon then assign sentiment label 'below average' to the identified aspect. If any sentiment shifter present, call sentiment shifter function.

Step 8: Go to the step 2 until all sentences of a feedback are processed.

Step 9: Neutral sentiment label 'average' is assigned to all other aspects not mentioned by the student in his / her feedback.

Step 10: Go to step 1 until all students' feedback are processed.

\subsection{Teacher Evaluation Reports}

This module is responsible to generate different customize reports on particular aspects or teacher. With this module we can compute overall effectiveness of teacher. This module permits to compare effectiveness of teachers with respects to others. We divide Teacher Evaluation Reports module in two sub modules i.e. Sentiment Aggregator and Report Generation. Functions of Sentiment Aggregator and Report Generation modules are explained below.

\subsubsection{Sentiment Aggregator}

This module is responsible for sentiment aggregation for all identified aspects for a particular teacher. Sentiment aggregation module reads sentiment status of each aspect as finalized by Aspect Sentiment Analyzer module corresponding to each student's feedback and generates aggregate score for each identified aspect. We compute value of Above_avg_agg, Below_avg_agg and Avg_agg for all the identified aspects using simple normalization formula.

Above_avg_agg (aspect) $=\frac{\text { Number of times aspect labeled as 'above average' in all feedbacks }}{\text { Total number of feedbacks. }}$

Similarly system compute value of Below_avg_agg (aspect) and Avg_ag (aspect). Above-avg_agg (aspect) represents fraction of students who think that a particular aspect is of above average level while Below_avg_agg (aspect) represents fraction of students who think that a particular aspect is of below average level and Avg_agg (aspect) represents fraction of students who think that a particular aspect is of average level. Values of Above-avg_agg (aspect), Below_avg_agg (aspect) and Avg_agg (aspect) will be in the range of 0 to 1 and sum of Above-avg_agg (aspect), Below_avg_agg (aspect) and Avg_agg (aspect) will be always 1 . Aggregation of 'Presentation' and 'Knowledge' aspects are shown below in Figure 2.
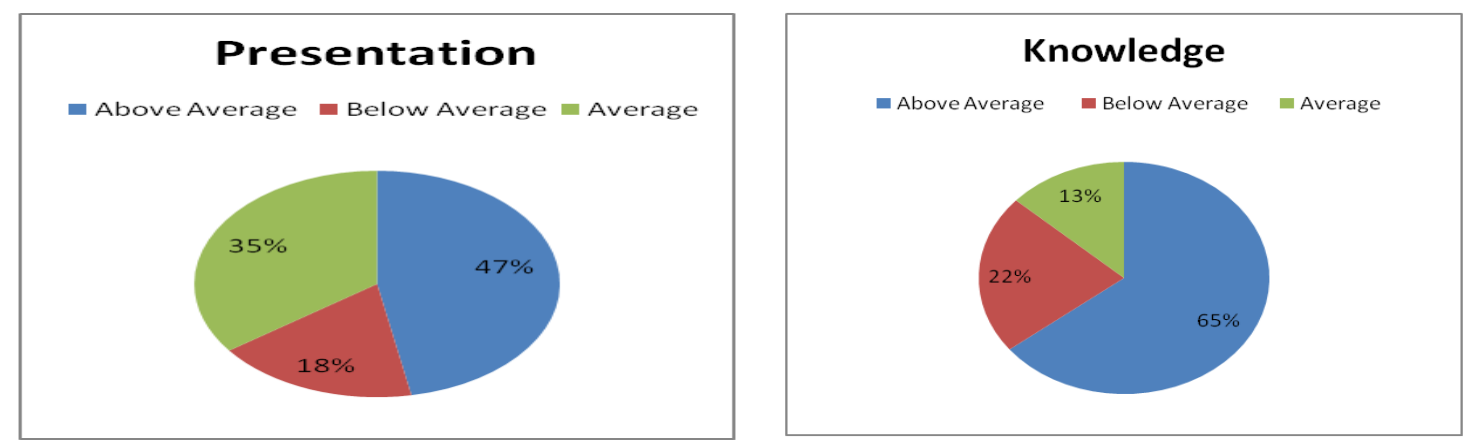

Figure 2. Aspect Aggregation

\subsubsection{Report Generation:}

With the help of this module, we can generate reports for a specific teacher to display computation results on all aspects or on some selected aspects. The following figure shows that particular teacher's knowledge, presentation, concepts and approach is of average level but he / she is not punctual and dedicated as shown in Figure 3. 


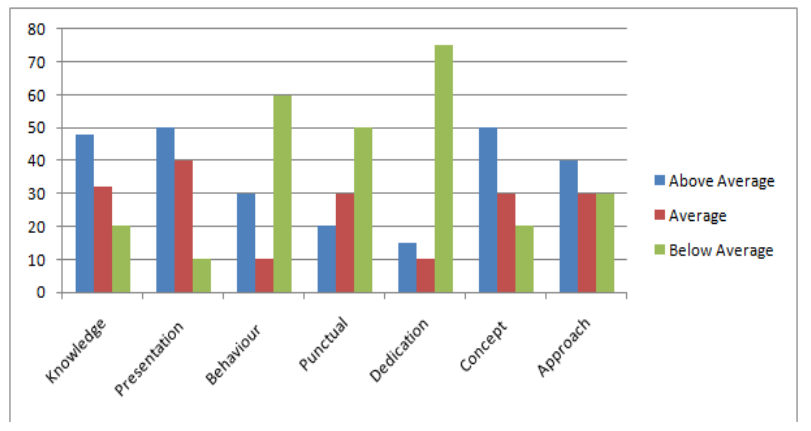

Figure 3 Teacher Feedback Report

Reports can also be generated to compare aspects of many teachers at the same time as shown in Figure 4. Figure demonstrate that Teacher- 2 is better than Teacher- 3 and Teacher- 1 is better than Teacher- 2 in most of the aspects.

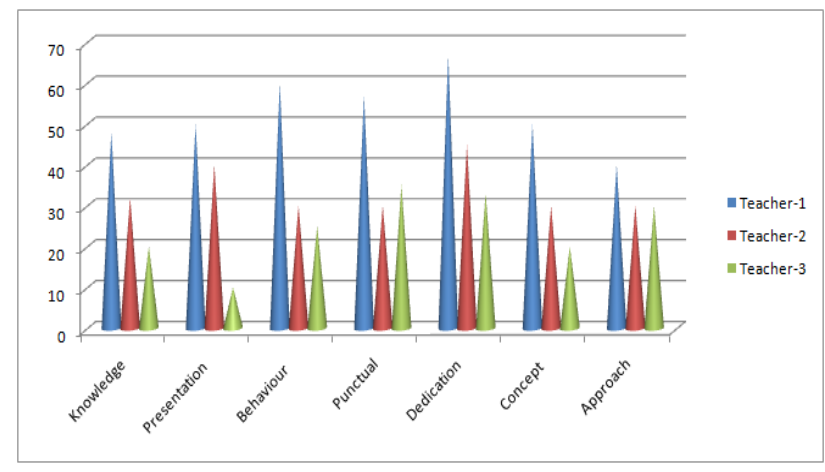

Figure 4. Two Teacher's Comparison Report

\section{RESULTS AND CONCLUSION}

Our opinion evaluation system allows us to extract all possible aspects of a teacher and then evaluate the teacher on each aspect. It can be seen that the system is able to identify many important aspects which are generally not mentioned in any questionnaire like 'supportive', 'biased', 'friendly', 'frank', etc. Table 1 shows the thirty most probable aspects identified by the Faculty Evaluation System (FES). The system allows us to compare one teacher with another teacher for all the chosen aspects.

Table 1. Aspects Identified by System

\begin{tabular}{ccc}
\hline Punctual & Assignment & paper pattern \\
\hline Disciplined & Strict & Teaching way \\
Intelligent & Explanation & Cooperative \\
Systematic & Paper pattern & Class Environment \\
Normal & Behavior & Frank \\
Teaching skill & Nature & Interesting \\
Approach & Interactive & Knowledge \\
Course & Bias & Supportive \\
Friendly & Behavior & Responsible \\
Concept & Sincere & Dedication \\
\hline
\end{tabular}

Faculty Evaluation System (FES) can be used by any organization to evaluate the utility of its employees in each area and can help in improving the productivity of its employees by making them aware of their weak points and allocating them more suitable work according to their strong points.

It is observed that opinion sentiment analysis of human beings needs to be handled differently than the sentiment analysis of product reviews due to some inherent properties of human beings. We need to incorporate more linguistic knowledge with machine learning algorithms to get good results and system 
accuracy can be enhanced by adding some job specific knowledge [21, 22]. We are in the process of implementing other machine learning algorithms at different stages of opinion evaluation system of human beings. This will allow us to compare our results and we will be able to use the most appropriate machine learning algorithms for Faculty Evaluation System.

\section{REFERENCES}

[1] Hu, Minqing and Bing Liu, "Mining and summarizing customer reviews," in Proceedings of ACM SIGKDD International Conference on Knowledge Discovery and Data Mining (KDD-2004), 2004.

[2] Popescu, Ana-Maria and Oren Etzioni, "Extracting product features and opinions from reviews," in Proceedings of Conference on Empirical Methods in Natural Language Processing (EMNLP-2005), 2005.

[3] Qiu, Guang, Bing Liu, Jiajun Bu, and Chun Chen, "Opinion Word Expansion and Target Extraction through Double Propagation," Computational Linguistics, Vol. 37, No. 1, pp. 9-27, 2011.

[4] Jin, Wei and Hung Hay Ho, "A novel lexicalized HMM-based learning framework for web opinion mining," in Proceedings of International Conference on Machine Learning (ICML-2009), 2009.

[5] Jakob, Niklas and Iryna Gurevych, "Extracting Opinion Targets in a Singleand Cross-Domain Setting with Conditional Random Fields," in Proceedings of Conference on Empirical Methods in Natural Language Processing (EMNLP-2010), 2010.

[6] Mei, Qiaozhu, Xu Ling, Matthew Wondra, Hang Su and ChengXiang Zhai, “Topic sentiment mixture: modeling facets and opinions in weblogs," in Proceedings of International Conference on World Wide Web, 2007.

[7] Alok Kumar and Renu Jain, "Sentiment Analysis and Feedback Evaluation," IEEE 3rd International Conference on MOOCs, Innovation and Technology in Education (MITE), 2015

[8] Ding, Xiaowen, Bing Liu, and Philip S., "Yu. A holistic lexicon-based approach to opinion mining," in Proceedings of the Conference on WebSearch and Web Data Mining (WSDM-2008), 2008.

[9] Liu, Bing, Sentiment Analysis and Subjectivity, in Handbook of Natural Language Processing, Second Edition, N. Indurkhya and F.J. Damerau, Editors. 2010.

[10] Bing Liu. Sentiment Analysis and Opinion Mining, Morgan \& Claypool Publishers, May 2012.

[11] Liu, Bing. Web Data Mining: Exploring Hyperlinks, Contents, and Usage Data, Springer, 2011.

[12] Liu, Bing, Minqing Hu, and Junsheng Cheng, "Opinion observer: Analyzing and comparing opinions on the web," in Proceedings of International Conference on World Wide Web (WWW-2005), 2005.

[13] Taboada, Maite, Julian Brooke, Milan Tofiloski, Kimberly Voll, and Manfred Stede, "Lexicon-based methods for sentiment analysis," Computational Linguistics, vol. 37(2), pp. 267-307, 2011.

[14] Kim, Soo-Min and Eduard Hovy, "Determining the sentiment of opinions," in Proceedings of International Conference on Computational Linguistics (COLING-2004), 2004.

[15] Riloff, Ellen, "Automatically constructing a dictionary for information extraction tasks," in Processing of AAAI2003. 1993.

[16] Qiu, Guang, Bing Liu, Jiajun Bu, and Chun Chen, "Expanding domain sentiment lexicon through double propagation," in Proceedings of International Joint Conference on Artificial Intelligence (IJCAI-2009). 2009.

[17] Blei, David, M. Andrew, Y. Ng, and Michael, I. Jordan, "Latent dirichlet allocation," The Journal of Machine Learning Research, 2003.

[18] Blair-Goldensohn, Sasha, Kerry Hannan, Ryan McDonald, Tyler Neylon, George A. Reis, and Jeff Reynar, Building a sentiment summarizer for local service reviews, in Proceedings of WWW-2008 workshop on NLP in the Information Explosion Era. 2008.

[19] Joachims, Thorsten, Making large-Scale SVM Learning Practical, in Advances in Kernel Methods - Support Vector Learning, B. Schölkopf, C. Burges, and A. Smola, Editors. MIT press, 1999.

[20] Shawe-Taylor, John and Nello Cristianini, Support Vector Machines. Cambridge University Press, 2000.

[21] Pang, Bo, Lillian Lee, and Shivakumar Vaithyanathan, "Thumbs up: sentiment classification using machine learning techniques," in Proceedings of Conference on Empirical Methods in Natural Language Processing (EMNLP-2002), 2002.

[22] Turney, Peter D., "Thumbs up or thumbs down?: semantic orientation applied to unsupervised classification of reviews," in Proceedings of Annual Meeting of the Association for Computational Linguistics (ACL-2002), 2002 